\title{
Competency-Based Education: A New Perspective on CSL Teacher Training Mode Construction
}

\author{
Wu CAI ${ }^{1, a,{ }^{*}}$ \\ ${ }^{1}$ Overseas Education College, Xiamen University, Xiamen, China \\ atotc1003@163.com
}

Keywords: Competency-based education, Core competence, CSL teachers, Training mode, Mainland China and Taiwan.

\begin{abstract}
Facing the issue of the shortage of qualified teachers, the construction of a more effective training mode for the teachers of Chinese as a second language (CSL) has become a hot topic. Competency-based education provides a new perspective and dimension for the training of CSL teachers. This paper outlines the development process and specific application of competency-based education. Meanwhile, the core competences of second language teachers are also presented. Then the author attempts to reveal the core competences of CSL teachers by integrating second language teachers' core competences with the assessment standards of CSL teachers both in mainland China and Taiwan. Finally, the CSL teacher training mode based on the principles of competency-based education is proposed.
\end{abstract}

\section{Introduction}

With the rapid development of international Chinese language education, the demand for excellent talents of international Chinese language education is also increasing. The shortage of the teachers of Chinese as a second language (CSL) has become a key issue which seriously restricts the spread of Chinese language. Whereas how to cultivate high-quality CSL teachers? The author believes that successful CSL teacher training should adapt to the social and marketable demand. However, in order to meet the needs of the society and market, what kind of competences should the CSL teachers have?

In the process of CSL teacher training, it is essential to make full use of all the teaching resources to maximize the effectiveness of teaching in the limited teaching time. In other words, the educators should try their best to make the pre-service CSL teachers obtain the core competences. Competency-based education provides a new perspective and method for the cultivation of excellent CSL teachers. The paper illustrates the development process and specific application of competency-based education. Then the author summarizes the core competences of CSL teachers on the basis of literature review and analysis. In the last part of this paper, the CSL teacher training mode based on the principles of competency-based education is proposed.

\section{Competency-Based Education and Its Application}

\section{The Development of Competency-Based Education}

The history of competency-based education can be traced back to the 19th Century. Brown believed that competency-based training was closely related to the competency movements [1]. He also discussed it in detail and divided it into five stages. The first generation of competency-based training reflected the application of scientific 
management to the workplace since the late 19th Century. The second generation of competency-based training supported the development of mastery learning which took place in the USA during the 1920s and 1930s. The third generation of competency-based training integrated psychology into the process of vocational education and training programs. The fourth generation of competency-based training had an obvious association with the performance-based teacher education movement during the 1960s and 1970s in the USA. The fifth generation of competency-based training emphasized learning outcomes which derived from the demand of work role.

After more than a century of development and evolution, competency-based education has become more and more mature and perfect. The curriculums based on competency-based education are designed after the analysis of prospective or actual role in labor market. In other words, according to the principles of competency-based education, the curriculums should be adapted to the actual needs of modern society.

It is not difficult to show the advantages of competency-based education. Some scholars put forward a series of reasons that could probably explain the popularity of competency-based education. For example, the development of competences can illustrate the positive goals of education and training clearly and competency-based education will bridge the gap between the demands of the job market and the traditional education [2]. Competency-based education also allows all students to study at their own pace, shorten the time required to graduate, reduce costs of learning and improve quality of education [3]. There is no doubt that competency-based education really brings a great impact on education and is of great significance to the educators.

On the other hand, competency-based education also has its limitations and shortcomings. For example, it is not easy to make agreement on the definition of competence. It is also difficult to observe and measure some kinds of competences, such as interpersonal communication, introspection and so on. The environmental, social and economic dimensions which competency-based education derives from are complex and dynamic [4]. Although competency-based education has its limitations, it does not affect its valuable application in education. On the contrary, recognizing the limitations of competency-based education can assist the educators to overcome its shortcomings, improve the effect of its application and make it serve education better.

\section{The Characteristics of Competence}

The characteristics of competence are very extensive. It brings a broad space for the application of competency-based education. When talking about the word of competence, different scholars have various definitions. The concept of competence can be both broad and narrow. Broadly speaking, competence is not only the application of knowledge and skills acquired in the past, but also effectively handles problems in an unknown environment and further achieves a deeper level. In a narrow sense, the concept of competency can be also applied in a particular field. For instance, the competence can be defined as a student's learning performance in a teaching program.

In addition, the meaning of competence is different in various situations. More specifically, the concept of competence can vary with different circumstances or perspectives. Van der Klink, Boon and Schlusmans illustrated the concept of competence from the views of geographical area, teaching theory and practice in detail [5]. From the perspective of geographical area, different countries have their own views. For instance, competence means behavioral and personality characteristics underlying possibly excellent performance in the United States. But it refers to the ability to perform the specified standards in advance in the United Kingdom. From the view of 
teaching theory, constructivism emphasizes the importance of conviction, motivation and ambition as the major aspect of competence. However, cognitive teaching theory focuses on teachable aspects of competence. Based on the view of practice, competence has its specific definition in acquisition, teaching, job evaluation and performance pay.

No matter in a broad sense or in a narrow sense and even in different environments, competence usually relates to knowledge, skill, or attitude. Building upon the extensive characteristics of competence, competency-based education implies more learning opportunities for students so that they can develop integrated, performance-oriented capabilities for dealing with different kinds of problems in different circumstances.

\section{The Application of Competency-Based Education}

With the rapid development of economy and society, especially the advent of the era of information, knowledge productivity has become the driving force in the twenty-first Century. The individual needs to develop the necessary capacity in order to better participate in the professional life based on knowledge and skill. On the basis of these considerations, the competences of graduates have become more and more important.

However, the traditional talent training model is difficult to meet the needs of society and labor market. It means the talents who are trained by higher education institutions should be able to have a strong adaptability and enough ability to face the complex and diverse society. Since the 1990s, a lot of educational institutions all around the world have begun to use the principles of competency-based education to make the teaching objectives, decide the teaching contents, design the teaching activities, develop the teaching strategies and evaluate the teaching effects. The educators expect to nurture high quality graduates who can meet the demands of employers and have the abilities to survive and develop in the post industry era and knowledge economy society.

It is obvious that competency-based education is a great innovation in the field of higher education. Nowadays, competency-based education has been applied to many countries and regions and has brought a profound impact. In Australia and the United Kingdom, competency-based education has become an important part of the national training reform program. In Europe, the European Higher Education Area recommends developing competences of graduates. Competence has become one of core elements in the European Qualifications Framework. In 1998, the educational department began to adopt the competency-based training program in the teacher education institutions in Flanders in Belgium. In the vocational education and training system in Holland, competency-based education is the dominant paradigm of innovation. In the United States, many curriculums in higher education institutions are designed by the framework of competence. Moreover, with the continuous development of modern educational technology, competency-based education has been integrated into different kinds of online curriculums. It further expands the scale of its application.

\section{CSL Teacher Training Mode Based on Competency-Based Education}

\section{The Core Competence of Second Language Teacher}

Competency-based education focuses on the development of students and is oriented by the industry need. Therefore, in order to better apply it to the CSL teacher training, the educators must first know what kind of competences should the CSL teachers have.

It is worthy of note that a successful CSL teacher is also a qualified second language teacher. The author reviews the related literatures and attempts to summarize the core 
competences of second language teacher. On the basis of literature review and serious analysis, the author finds that besides the enough language competence, the other core competences of second language teacher still include the following categories.

Intercultural Competence. With the rapid development of globalization, intercultural competence is particularly important for second language teachers in the 21st Century. The second language teachers have to be equipped with intercultural perspective to accomplish various tasks in appropriate ways. Therefore, there has been an increasing awareness of developing the intercultural competence of pre-service second language teachers. On the other hand, in the process of second language teaching, it is imperative for teachers to help learners to acquire the knowledge and skills of the target language and develop cultural awareness at the same time. For achieving these goals, the teacher education programs should offer more opportunities to the pre-service teachers to go abroad for training. It is helpful for the pre-service teachers to develop an intercultural approach in the second language teaching practice.

Intercultural competence is also a complex and pluralistic concept. Byram believed that intercultural competence in the field of foreign language education consists of four dimensions: knowledge, attitude, skill and critical cultural awareness [6]. Sercu also pointed out it is necessary for foreign language teachers to be familiar with the foreign cultures associated with the foreign languages they teach [7]. In a word, Nurturing intercultural competence is an indispensable part of second language teacher training.

Information and Communications Technology Competence. With the rapid development of information and communications technology, computerization has become one of the dominant trends in the modernization of the educational system all over the world. Meanwhile it poses a great challenge to the second language teachers in the complex and dynamic educational environment. In other words, in order to meet the needs of the times and achieve better teaching results, the second language teachers are required to apply information and communications technology in various courses.

In the recent years, the cultivation of the competence of information and communications technology of second language teachers has been advocated by more and more scholars. Malinina put forward information and communications technology was of great value to foreign language education [8]. He believed that it could increase transparency, convenience and flexibility of education. It promotes autonomy and cooperation of learners, provides effective learning experience and presents sufficient learning information too. Information and communications technology is also of great help to find an appropriate teaching and learning method because of its fast feedback.

Some scholars attempt to create the model of digital competence or the framework of technological pedagogical and content knowledge (TPACK) in the second language teacher education and training. For example, Røkene and Krumsvik proposed the model of digital competence for the student teachers of English as a second language (ESL). It contains basic digital skill, didactic ICT-competence, learning strategies and digital bildung [9]. In order to achieve better teaching effect, Cai pointed out CSL teachers should apply the ICT knowledge, skills and resources appropriately in teaching Chinese as a second language [10]. He gave some suggestions on developing pre-service CSL teachers' TPACK in the teacher training. As the high pace of the development of information technology, second language teachers' information and communications technology will play an increasingly key role in the teaching practice in the future.

Pedagogical Competence. Second language education is an interdisciplinary field which needs a great number of complex teaching methods. For a second language teacher, how to shape excellent teaching competence is the key to the success of 
teaching. Madhavaram and Laverie defined pedagogical competence as "the ability of an individual to use a coordinated, synergistic combination of tangible resources and intangible resources to achieve efficiency and/or effectiveness in pedagogy" [11]. It fully expressed the connotation of pedagogical competence. Mâță, Cmeciu and Ghiațău classified the pedagogical competence of language teachers into three categories which are cognitive component, behavioral component and attitudinal component [12]. It reflected that in the field of language teaching, teaching contents includes knowledge, skills, attitudes and values which make the pedagogical competence more complicated.

\section{Competency-Based CSL Teacher Training Mode Construction}

Besides the language competence, intercultural competence, information and communications technology competence and pedagogical competence, CSL Teachers also have their own characteristics of competence. In recent years, both sides of the Taiwan Strait have cultivated lots of CSL teachers and both play important role in the international Chinese language education. Integrating the core competences of the second language teachers with the assessment standards of CSL teachers in mainland China and Taiwan, the author attempts to reveal the core competences of CSL teachers.

In mainland China, the Office of Chinese Language Council International has formulated and revised the Standards for Teachers of Chinese to Speakers of Other Languages. Now it contains five standards. The first standard is the basic knowledge in Chinese teaching. The second one is the teaching methods of Chinese language. The third one is the teaching organization and classroom management. The fourth one is the Chinese culture and intercultural communication. The last one is the professional ethics and development. In Taiwan, there are also five parts in the certification examination for proficiency in teaching Chinese as a second language. They are Chinese, Chinese society and culture, spoken Chinese and expression, teaching Chinese as a second language and Chinese Linguistics. They are both the important criteria of CSL teachers.

After comparing and analysis, we can find the core competences of CSL teachers: (1) Chinese and foreign language knowledge and skills; (2) intercultural competence (including the ability to understand and compare Chinese culture and foreign cultures); (3) acquisition strategy of Chinese as a second language; (4) Pluralistic teaching methods (e.g. the integration of information technology); (5) professional ethics and psychological quality; (6) classroom management, adaptability and interpersonal skills.

On the basis of these competences, we can establish a competency-based training model of CSL teacher training. It consists of the following steps. First of all, confirming the core competences of CSL teachers; in the second, setting up the course objectives which are correspond with the core competences of CSL teachers; and third, carrying out the rich and effective teaching activities; in the end of the teaching mode, adopting multiple evaluation methods after the implement of all the teaching activities and tasks.

It is obvious that this training mode of CSL teacher training is based on the core competences of the qualified CSL teachers. And the core competences derive from the actual demand of teaching and the needs of job market and society. Therefore the educators develop curriculum objectives and carry out teaching practice centered on the nurturing of CSL teachers' expected capacities. In particularly, the final evaluation criterion is multiple and does not only focus on the scores of students. The key of assessment lies in whether the students achieve the goals which are designed in advance and acquire the expected core competences of CSL teachers. It will absolutely be different from the traditional training modes and bring a great impact on the CSL teachers training. 


\section{Summary}

Competency-based education makes the link between the social needs and the students. It enhances the students' learning initiative and motivation. In the CSL teachers' training, it also provides a new perspective for the cultivation of CSL Teachers. In the recent years, mainland China and Taiwan have both faced the problem of the shortage of qualified teachers. This paper summarizes the core competences of CSL teachers and proposes the mode of CSL teacher training based on competency-based education.

It is hoped that in the future, there will be an appropriate opportunity to conduct an empirical study on the CSL teachers in mainland China and Taiwan. It will be of great help to further revise and subdivide the competence indicators to make the core competences of CSL teachers and teacher training mode more scientific and reasonable.

\section{References}

[1] M. Brown, An introduction to the discourse on competency-based training (CBT), in: M. Brown et al., (Eds.), A Collection of Readings Related to Competency-based Training: EAE604 Curriculum and Competences, Deakin University, Geelong, 1994, pp. 1-17.

[2] K. Struyven and M.D. Meyst, Competence-based teacher education: Illusion or reality? An assessment of the implementation status in Flanders from teachers' and students' points of view, Teaching and Teacher Education 26 (2010) 1495-1510.

[3] Information on

http://www.huffingtonpost.com/dr-robert-mendenhall/competency-based-learning-_b_ 1855374.html.

[4] J.F. Schilling and J.R. Koetting, Underpinnings of competency-based education, Athletic Train Education Journal. 5 (4) (2010) 165-169.

[5] M. van der Klink, J. Boon and K. Schlusmans, Competences and vocational higher education, Now and in Future European Journal of Vocational Training 40(2007)67-82.

[6] M. Byram, Teaching and Assessing Intercultural Communicative Competence, Multilingual Matters, Clevedon, 1997.

[7] L. Sercu, The foreign language and intercultural competence teacher: The acquisition of a new professional identity, Intercultural Education, 17 (1) (2006) 55-72.

[8] I. Malinina, ICT competences of foreign languages teachers, Procedia- Social and Behavioral Sciences. 182 (2015) 75-80.

[9] F.M. Røkene and R.J. Krumsvik, Prepared to teach ESL with ICT? A study of digital competence in Norwegian teacher education, Computers \& Education. 97 (2016) $1-20$.

[10]W. Cai, TPACK: A new dimension to Chinese as a second language teacher training, Proceedings of the 2016 2nd International Conference on Social Science and Higher Education (ICSSHE 2016), Advances in Social Science, Education and Humanities Research. 53 (2016) 460-465.

[11] S.Madhavaram and D.A. Laverie, Developing pedagogical competence: Issues and implications for marketing education. Journal of Marketing Education. 32 (2) (2010) 197-213. 
[12]L. Mâță, D.Cmeciu and R.M. Ghiațău, A reference framework of pedagogical competences of language teachers in the initial training programmes, Procedia - Social and Behavioral Sciences. 93 (2013) 648 - 653. 